\title{
Preliminary design of a VTOL unmanned aerial system for remote sensing of landscapes
}

\begin{abstract}
In this research defines the preliminary design - synthesis of a general visual inspection system of quadrotor type, for remote sensing of landscapes. It was shown that as a flying vehicle, the quadrotor has respectable place among vertical take-off and landing vehicles for this purpose. Was made definition unmanned aerial system configuration according design constraints. After analysis was proposed and described unmanned aerial system, selected for this request. For chosen system was made close-range camera calibration, studied image features such as Harris Corners which can serve as a compact image representation for remote sensing of landscapes.
\end{abstract}

Keywords: vertical take-off and landing (VTOL), unmanned aerial system (UAS), remote sensing of landscapes (RSL), quadrotor

\author{
Volume 4 Issue 2 - 2020
}

\author{
Nickolay Zosimovych \\ Intelligent Manufacturing Key Laboratory of Ministry of \\ Education, Shantou University, Shantou, China
}

Correspondence: Nickolay Zosimovych, Intelligent

Manufacturing Key Laboratory of Ministry of Education, Shantou

University, Shantou, China, Tel +8613794104463,

Email nzosimvych@gmail.com

Received: June 04, 2020 | Published: June 26, 2020

\section{Introduction}

We are a visual human, as mentioned J. Adams, ${ }^{1}$ that depend on deeply on images to recognize the world about us. The competence of people to abstract data from landscapes over visual descriptions has changed over many million years on our planet. Aerial photos and satellite images permit us to envision landscapes in completely novel behaviors. We can see immense ranges all at once. We can construct images that measure diverse properties of landscapes, and we can compare images engaged at different times to discover changes. The power of these remote measurements based in the fact that we can relate them to the familiar realm of what we see on the ground. Remote sensing is not just an exciting practical miracle; it has developed a vital part of a crucial mission to recognize our varying environment. ${ }^{1}$

Remote Sensing of Landscapes (RSL) is a mutual method of quality control, data gaining and data analysis, concerning raw human senses such as vision, hearing, touch, smell, and/or any nonspecialized inspection equipment. Modern unmanned aerial systems (UAS), usually called as unmanned aerial vehicles, equipped with the suitable payload and sensors for explicit tasks, are being established for automated visual examination and monitoring in many urban, industrial, rural, and agricultural applications.

In remote sensing, as Q. Weng wrote, the sensors are not in direct contact with the objects or events being observed. Moreover, electromagnetic radiation generally is used as the information shipper in remote sensing. The output of a remote sensing system is typically an image demonstrating the sight being detected. An additional step of image analysis and interpretation is necessary to remove useful information from that image. In a more limited sense, remote sensing states to the science and technology of gaining data about our planet surface (land, lakes, rivers, urbans, and ocean) and atmosphere using sensors onboard airborne (UAS, aircraft, balloons, rockets) or space borne (satellites and spacecraft's) platforms. Dependent on the opportunity, remote sensing may be split into next categories: ${ }^{2}$ 1) satellite remote sensing; 2) photography and photogrammetry; 3) thermal remote sensing (thermal infrared spectrum); 4) radar remote sensing (microwave wavelengths); and 5) LiDAR remote sensing (laser pulses are transmitted to the ground and the space between the sensor and the ground is measured established on the return time of each pulse).
Regardless of passive or active remote sensing systems, all sensing systems detect and record vitality signals from earth surface features and/or from the atmosphere. Acquainted samples of remote sensing systems comprise aerial cameras and video recorders. More intricate sensing systems contain electronic scanners, linear/area arrays, laser scanning systems, etc. Data composed by that remote sensing systems can be in also analog format (hardcopy aerial photography or video data) or digital format (usual radiance measured within an image pixel). Digital remote sensing images may be response directly into a GIS for use, like analog data. ${ }^{2}$

Remote sensing satellites help us in a number of ways to study natural resources, vegetation forms and agrarian crop production trends. But despite great steps in the complexity of equipment, modern computer aided processing and multispectral images, the satellite-aided mapping of crop production regions is still not an easy task, as was mentioned in Ref. ${ }^{3}$

In-flight operations have been operated as an alternative to satellites as these offer high resolution photography. Such images are useful to agencies commerce with natural resource monitoring, as marked K.R. Krishna in his book. Researcher wrote that airborne remote sensing offers shorter turn around and helps in initiating aerial photography, at short notice and as many times as required. Moreover, the spectral resolutions of airborne campaigns have ranged from 0.5 to $2.0 \mathrm{~m}$ pixel sizes with $2-20 \mathrm{~nm}$ bandwidths in the $450-2500$ $\mathrm{nm}$ spectral range. Such aerial images can be employed in revising vegetation, crop supervision, estimating leaf chlorophyll contents and detecting drought-affected regions. And the airborne visible infrared imager provides information on leaf water potential. ${ }^{3}$ But the main disadvantage of that systems still high expenses.

Modern tendency in monitoring natural resources, vegetation and agrarian bindings is to accept UAS, that have mini sensors. They are rapid in turn around and offer very high resolve images. They are really capable of undertaking a wide kind of tasks associated to remote sensing of landscapes. Main applications of UAS with concern to natural resource monitoring, are next: ${ }^{3}$ 1) topographic mapping; 2) capture of images of natural features and other structures; 3) analysis of natural vegetation; 4) water level mapping; and 5) mapping environmental effects on soil resources etc. 
Ref. $^{3}$ defines the preliminary design - synthesis of a general visual inspection (GVI) for RSL system onboard a profitable VTOL (Vertical Take-Off and Landing) UAS, and namely, quadrotor type. The experimental setup is composed of a ground station (based on laptop), an embedded control system installed on the airframe, and sensors dedicated to GVI processing during remote sensing. A highdefinition onboard camera will spot visual harm produced by hail or lightning strikes, which are between the most hazardous threats for the airframe. ${ }^{4}$ The proposed hovering UAS will be pushed along the fuselage and wings, and the vehicle automatically maintains a safe standoff distance from the aircraft due to ultrasonic sensors.

\section{The design process background}

The design of most existing UAS systems, usually will be considered to begin in three phases: ${ }^{5} 1$ ) the conceptual phase; 2) the preliminary design phase; 3 ) the detail design phase. Other phases back after initial manufacture, assembly, and tests. These comprise the design of modifications throughout development and successive variations or developments whereas the UAS is in facility. As think R. Austin, techniques of operational analysis, cost-benefit and economic studies should be used namely during conceptual phase. It may be decided that the scheme is only possible if convinced new equipment is supported. This may put on, of course, to any of the elements of the system, for example, in air vehicle control or navigation, or in computation, communications or displays. Therefore, a phase of research may be directed and the conclusion to keep or not with the project will expect the result. $^{5}$

During next phase, called preliminary design, the original outline design of the whole structure will be stretched in more detail. Optimization trade-offs inside the system will be completed to maximize the total performance of the system over its estimated working roles and atmospheric conditions. Furthermore, a UAS mock-up and operator parts of the control station may be created to give a well obligation in three dimensions as to how components will be attached relative to one another, simply of availability for maintenance and operator ergonomics, etc. This facility, as was mentioned in, ${ }^{5}$ is becoming fewer essential, with the accessibility of $3 \mathrm{D}$ computer design soft. However, the physical obligation attainable from existing hardware should not be rejected casually. Moreover, it will be determined which elements of the system will be manufactured 'in house' and which will be procured, at what estimated cost, from alternative outside contractors.

The preliminary design phase achieves with an all-inclusive design meaning of the complete system with its interfaces and a system requirement. The price of the residual phases of the project and the costs of system operation will have been re-examined in better factor and the choice to proceed advance should be reconsidered. Therefore, costs will have been comparatively small. It is attractive for the programme management to wish over-hasty conclusion of that phase, but sometimes it could be a false economy. ${ }^{5}$

At detail design phase the work involved expands and a larger quantity of staff will be employed on the UAS project. Currently, a wide-ranging of UAS exist, from very small and lightweight fixed-wing aircraft to rotor helicopters, large-wingspan airplanes and quadrotors, each one for a specific task, normally providing determination outside the competences of manned vehicles. ${ }^{4}$ UAS for remote sensing of landscapes can be classified with respect to mass, range, flight altitude, endurance and etc. (Table 1). For the modeling, we used real parameters of the stabilization system of the simulated automatic interplanetary station discussed previously.

Table I Classification UAS for remote sensing of landscapes

\begin{tabular}{lllll}
\hline Category & Mass, $\mathbf{k g}$ & Range, $\mathbf{k m}$ & Altitude, $\mathbf{m}$ & Endurance, $\mathbf{h}$ \\
\hline Micro UAV & $0 \ldots 5$ & $0 \ldots 10$ & $0 \ldots 250$ & $0 \ldots 1$ \\
Mini UAV & $5 \ldots 25$ & $0 \ldots 10$ & $0 \ldots 300$ & $0 \ldots 2$ \\
Low altitude and long endurance & $25 \ldots 1500$ & $500 \ldots 2000$ & $0 \ldots 3000$ & $24 \ldots 48$ \\
Medium altitude and long endurance & $1000 \ldots 1500$ & $500 \ldots 2000$ & $0 \ldots 3000$ & $24 \ldots 48$
\end{tabular}

Practice show that VTOL aircrafts offer many advantages over conventional take-off and landing flying vehicles, mostly due to the small range required for take-off and landing, and the ability to hover in place and fly at very low altitudes and in narrow, limited spaces. In the core of VTOL aircrafts, such as conventional helicopters and crafts with rotors like the tiltrotor and fixed-wing aircraft with directed jet thrust ability, the quadrotor, or quadcopter, is very often selected, especially in scientific investigation on mini- or micro-size UAS, as an effective alternative to the high cost and complexity of conventional rotorcrafts, due to its capacity to hover and move without the complex system of connections and blade elements present in single-rotor vehicles. $^{6-8}$

Now a day quadrotors are broadly used in close-range photogrammetry, search and rescue operations, environment monitoring, industrial component inspection, etc., due to their rapid maneuverability in small areas. As a flying vehicle, the quadrotor has respectable place among VTOL vehicles. However, it has some disadvantages. And first of all, as show publications [4, 7], the drone size is fairly bigger, energy consumption is larger, inferring minor flying time, and the control algorithms are very complex due to the fact that only 4 actuators are used to control 6 degrees of freedom of the quadrotor, and that the varying aerodynamic interference shapes among the rotors have to be taken into account.

\section{Definition VTOL configuration for remote sensing of landscapes}

Let us start from formulating the VTOL system design constraints: ${ }^{9}$

a. The propulsion system should have take-off point to be at the $50 \%$ throttle mark.

b. The system should carry a payload of up to $3 \mathrm{~kg}$.

c. The system should have a thrust to weight ratio within the range of $2: 1 \ldots 3: 1$. 
d. The system total mass must be $<7 \mathrm{~kg}$ (according to many countries drones flight regulations).

e. The system should be use a $6 \mathrm{~S}(22.2 \mathrm{~V})$ Li-Po power supply (because efficiency increases with voltage).

f. The system should be portable and occupy the minimum possible space.

g. Designer must take into account system failsafe measures, such as link loss, low battery voltage, GPS loss, etc.

h. The system should be low cost, robust and reliable.

Moreover, a distinctive rate for the maximum endurance of a small VTOL system for Remote Sensing of Landscapes $(<7 \mathrm{~kg})$ is about $20 \mathrm{~min}$. However, the greatest advantage has an endurance of around $30-40 \mathrm{~min}$ (usually quoted without payload in this case). ${ }^{9}$ The ultimate goal, as said Mr. S.D. Prior in ref. ${ }^{9}$ should be to exceed the 1 $\mathrm{hr}$ endurance target, whilst carrying a payload like $5 \mathrm{~kg}$, which would cover $90 \%$ of all current payloads. To achieve that, would require a system design at around $15 \mathrm{~kg}$, perhaps utilizing a more exotic power source such as a Hydrogen Fuel Cell, Fuel Engine or Hybrid system. Various commercial VTOL system may carry a small camera, which typically forms their payload. Enhancements to that device have allowed high definition cameras, with recording capability, to be unified into small, lightweight $(<30 \mathrm{~g})$ and low-cost packages. ${ }^{9}$ So, the goal of VTOL configuration for remote sensing of landscapes must be maximize as endurance, as payload capacity.

The mission demands that onboard electronic speed controller should be very high efficiency $(98 \%)$, low resistance (naturally 0.01 $\Omega$ ), high current switch. That component takes the control input from the autopilot and converts that to a three-phase signal which can be control the speed of the motor output shaft (prop). So, many electronic speed controllers similarly combine a battery elimination circuit to regulate a voltage $(5 \mathrm{~V})$ to power the receiver or another device, removing the need for an isolated Li-Po battery for this purpose. Additionally, they are restricted to a few Amps at most, so designer must be careful and not to overload them by asking them to drive large servos! In ref. ${ }^{9}$ caution that electronic speed controllers are generally lightweight and low cost. However, they are prone to overheating, which can result in a thermal shutdown causing a VTOL crash scenario, if pushed too hard or incorrectly indicated during connection.

The universal brushless DC (BLDC) motor has the rotor on the outside, incorporated into the casing. This is opposite to the older system design. The BLDC motor is basically a standard DC motor, without the old carbon brushes, which caused friction, sparks and ultimately failure due to wear. However, there are three windings and therefore this is classified as a three-phase BLDC motor, similar in nature to a synchronous AC motor. As mentioned, Mr. S.D. Prior in his book, ${ }^{9}$ the recent generation of electronic speed controllers use a pretty simple scalar form of control, like six-step commutation, with current being passed through only two of the three windings at any one time. The latest innovation in the VTOL systems design is by installing a vector control strategy, named field oriented control, which claims higher torque, less torque ripple and faster response rates (Figure 1).

The UAS designer may selects the basic configuration and then the individual propulsion elements from a series of drop-down online menus. However, practical designer does have the option of manually entering their own data based on measurements, if the proposed component is not available to select. ${ }^{9}$ Today, the good thing about UAS software packages are available online for a fairly low cost.

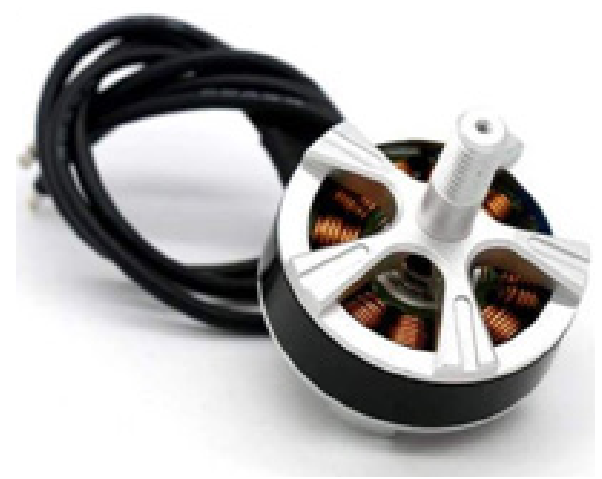

Figure I Brushless DC Motor NFP-BL-2204. ${ }^{10}$

Another very important thing is a propeller or rotor in a rotarywing vehicle is equivalent to the wing of a fixed-wing aircraft. The propeller (rotor) converts the rotating output power from the BLDC motor into the movement of air using the principle of Newton's 3rd Law of Motion. For now, universal propellers used in small multirotors variety from 1 " to 40 " in diameter, with 9-18" presence the most shared. ${ }^{9}$ The Figure 2 shows that even props with the same diameter and pitch can have varying efficiencies, ranging from $10 \%$ to $72 \%$ depending propellers diameter. So, the UAS propeller efficiencies of $0.7-0.8(70-80 \%)$, modelled in Figure 2, very nice correlate with experiment data, close enough as in ref, ${ }^{9}$ and are considered to be satisfactory.

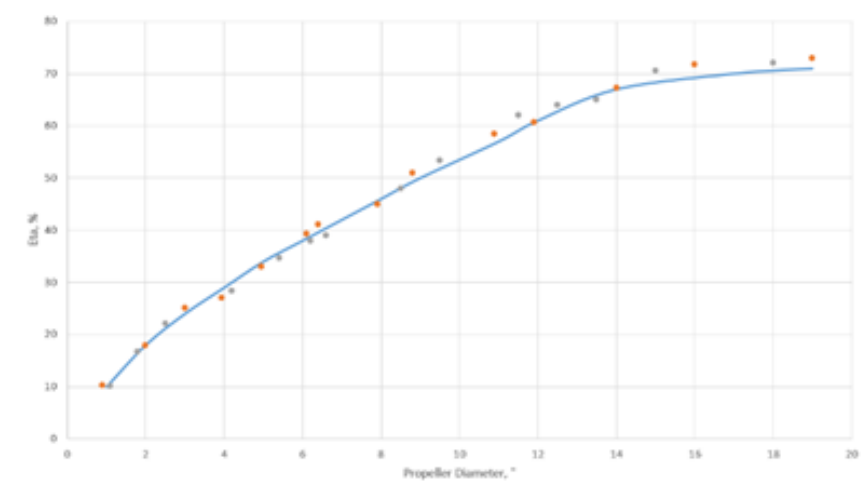

Figure 2 Propeller efficiency characteristic depending with its diameter.

Figure 3 represent the characteristics of motor efficiency from angular velocity for 3 different BLDC motors. Characteristics changes linearly below their maximum efficiencies. Figure 4 represent the characteristics of BLDC output power and thrust from angular velocity. All this characteristics, shown in Figure 2-4 have to be put for preliminary design data.

It should be remarks that any engineering design process, and our preliminary design of an VTOL for Remote Sensing of Landscapes is not exception too. So, designers may use in practice next main steps of preliminary design:
a. Define VTOL configuration.
b. Propulsion system design.
c. Component selection.
d. Constraints and requirements. 
e. Operation concept.

f. Redefine VTOL configuration or stop design process.

And we must to remember that every preliminary design process is iterative to get the most suitable option.

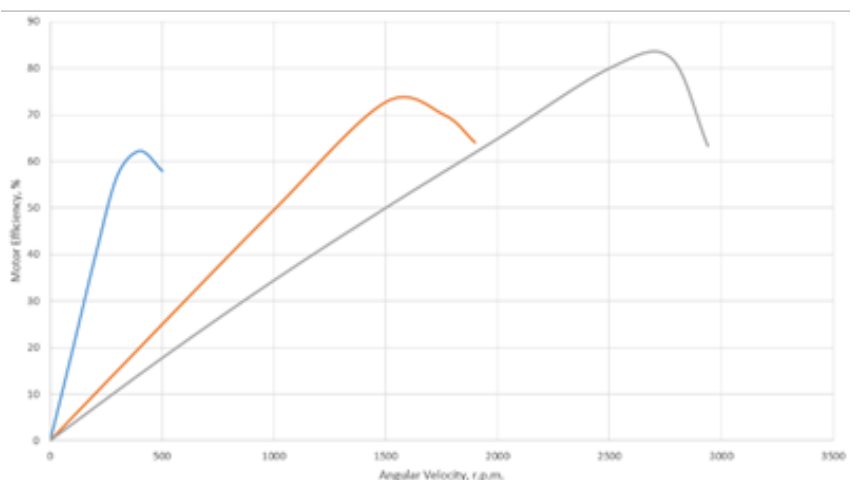

Figure 3 Characteristics of motor efficiency from angular velocity (for 3 different BLDC angular velocity motors).

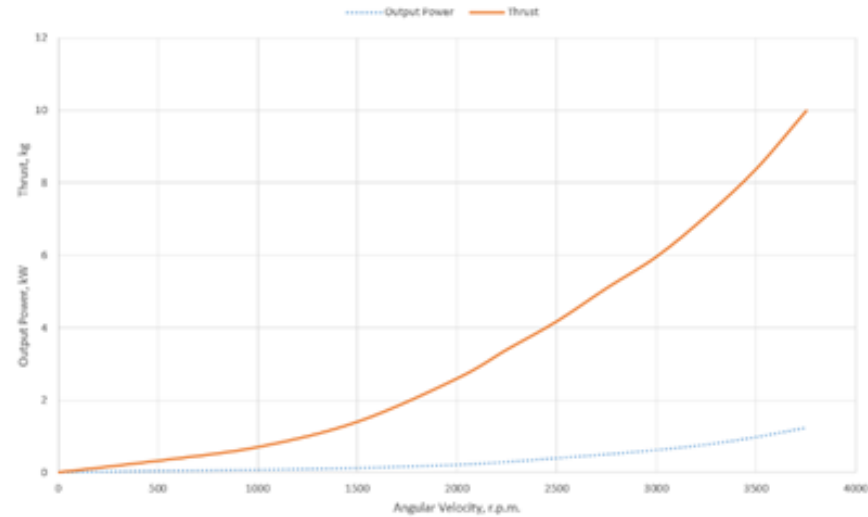

Figure 4 Characteristics of BLDC output power and thrust from angular velocity.

\section{VTOL unmanned aerial system example for remote sensing of landscapes}

As a rational example of VTOL unmanned aerial system selected for this request is a micro-UAS (Table 1) quadrotor, drone "Holy Stone HS700D FPV" (Figure 5), made by RC logger. ${ }^{11}$ Product dimensions are next: $15.2 \times 15.2 \times 6.1$ inches (without protection guards); total weight: $630 \mathrm{~g}$; and a payload capacity of $150 \mathrm{~g}$. The high-capacity $2800 \mathrm{mAh}$ intelligent battery yields up to $22 \mathrm{~min}$ flight time. It also has low-power alert. You can immerse yourself in a substantial flight. The onboard camera $\left(2048^{*} 1152 \mathrm{P}\right)$ with $110^{\circ}$ FOV (field of view) lens gives a panoramic view for recording your impressive moments. $5 \mathrm{G}$ broadcast confirms fast and high-quality FPV real time video over 1300 FT. Edit and share your new creations to applied resources or in proper media with your drone app directly. With GPS location, that drone can hover steadily and automatically return to home place precisely when its battery is low or the signal is lost. ${ }^{11}$ According to the task, you may provide professional RSL with easiness when the drone can follow you or fly in a circle along the path you set.

All of this made HS700D FPV drone as one of the most suitable VTOL system for remote sensing of landscapes. This means you can modify and choose the quality of resolution as you need in practice. Suitable even for the majority of beginner's researchers and of course, to take some really good aerial shots. ${ }^{13}$

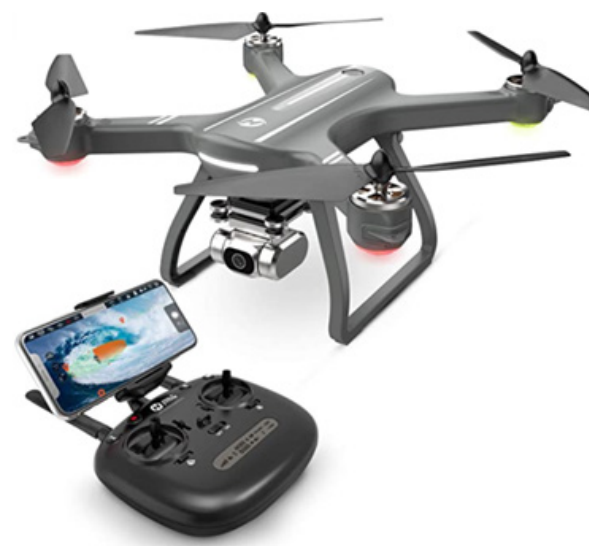

Figure 5 HS700D FPV drone with FHD camera FPV live video, GPS RC with brushless motor, $5 \mathrm{G}$ transmission, and auto return home.

To guarantee control and thrive all the flying stages during RSL term, a distance acquisition system using sonic ranging sensors was choosing, installed and tested as in laboratory as in-flight options. The image acquisition subsystem created for the RSL equipment contains of a Dorhea Raspberry Pi4B3B + Camera Module, released in spring 2020 (Figure 7), supported with one of the latest versions of Raspberry Pi's preferred operating system. ${ }^{13}$ For today, all current models of Raspberry Pi computer have a port for connecting the camera module (Figure 8). ${ }^{14}$ The entire weight of the image acquisition hardware (including with close-camera, computer module and case) is $48 \mathrm{~g}$. This unit allows manual control of the focal length, in order to set up specific and constant values. It can get images with a maximum resolution of about 6 Megapixels at a maximum rate of 90 frames per second with various picture formats like JPEG, RAW, GIF, BMP, PNG, YUV420, RGB888 (Figure 8).
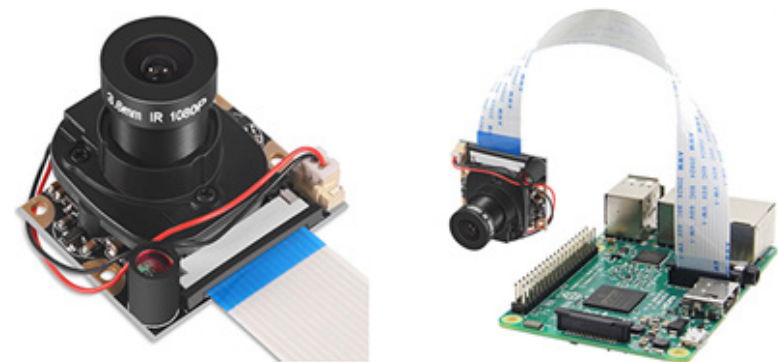

Figure 6 Dorhea Raspberry Pi4B3B+ camera module with Raspberry Pi2/3 Model B.

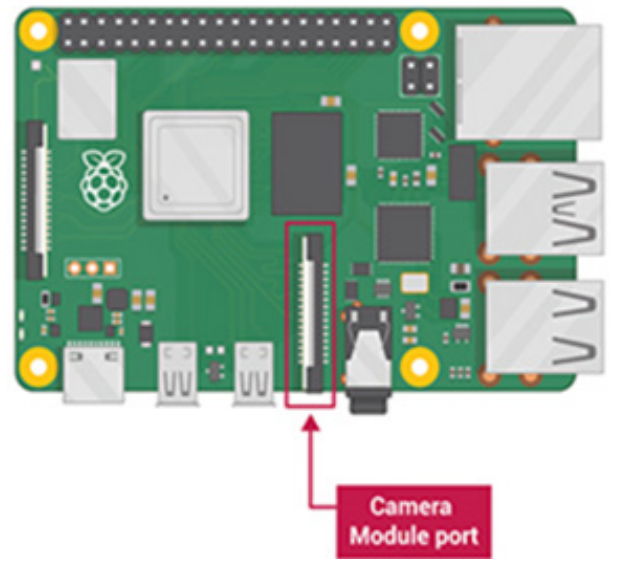

Figure 7 Raspberry Pi computer with a camera module port. 

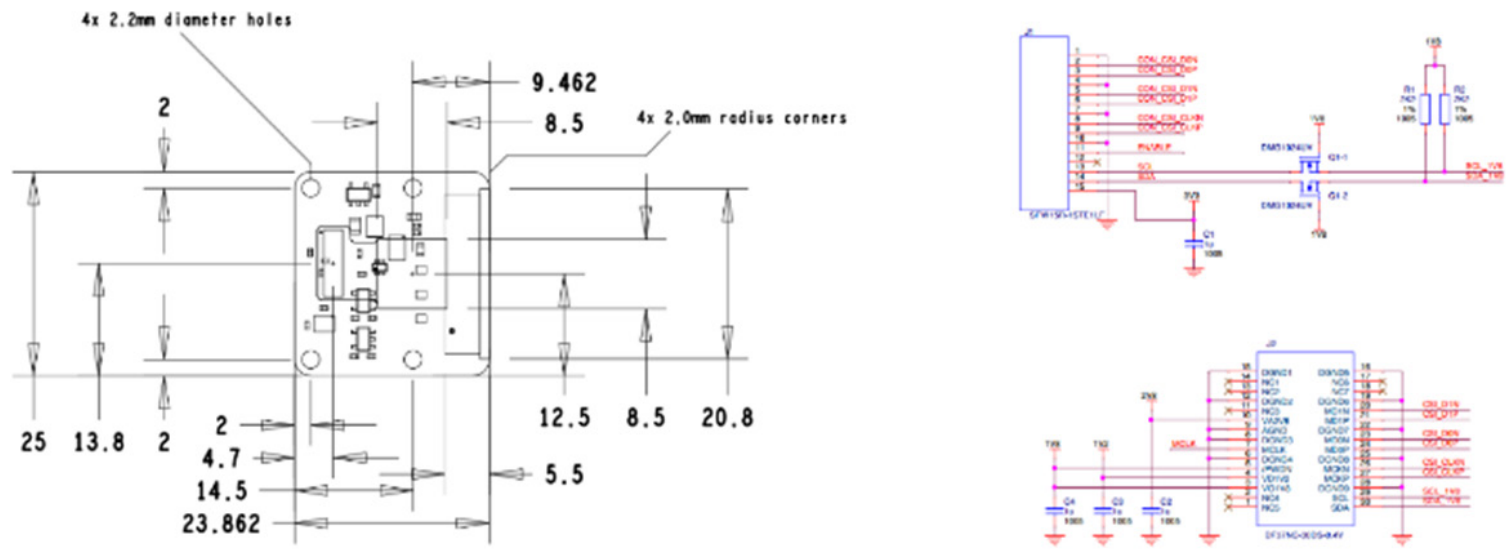

Figure 8 Drawings of a Rapsberry PI camera module V2.I and its electric schematics. ${ }^{14}$

\section{Harris corner detector study}

Onboard close-range camera calibration, or the procedure that permits defining the camera inner orientation and the distortion coefficients, has been done in the MATLAB environment and using some coded targets. ${ }^{4}$ The primary point of image center, the effective focal length and the radial distortion coefficients were projected to account for the difference of lens distortion within the field of view, and to determine the location of the onboard camera.

The Hough transform has been chosen due to its efficiency for its real time automatic recognition and since it is existing in the OpenCV library and customizable in the Python environment. The visual inspection by using Hough transform-processed images to highlight possible harm, enclosed by a circular or rectangular area. It was applied the image processing algorithm, developed by U. Papa and S. Ponte ${ }^{4}$ and detached to the embedded onboard computer of the proposed UAS, basically loads and blurs the image to reduce the noise. But, in this research was studied image features such as Harris Corners can serve as a compact image representation useful for task such as image matching, computing image statistics, 3D model estimation and video tracking during real-time RSL.

In first experiment was built Harris Corner Detector (Figure 9). It was writing a function to filter a grayscale image with a Gaussian filter. Subsequent objectives were required: 1) derive image gradients; 2) computer the corner score for each image pixel and 3) perform nonmaximum suppression and thresholding to isolate the image locations with the strongest corner scores.
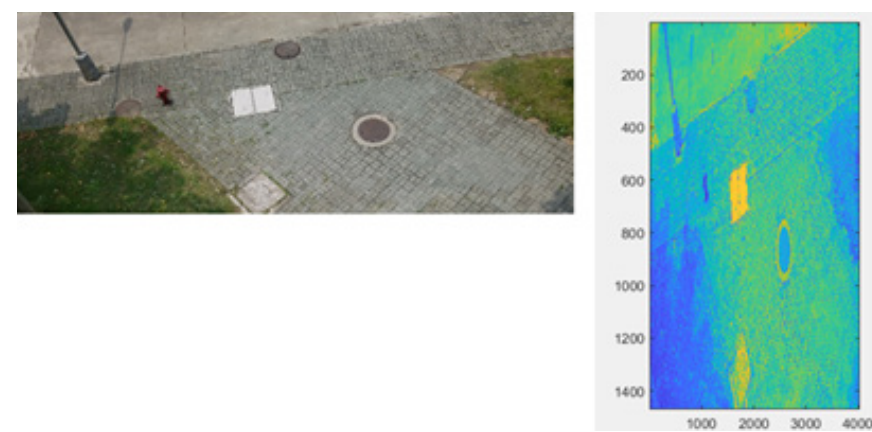

Figure 9 Original picture and grayscale image with a Gaussian filter.

In second experiment was compute image gradients. In this case image features such as Harris Corners can serve as a compact image representation useful for task such as image matching, computing image statistics, 3D model estimation and video tracking (Figure 10).

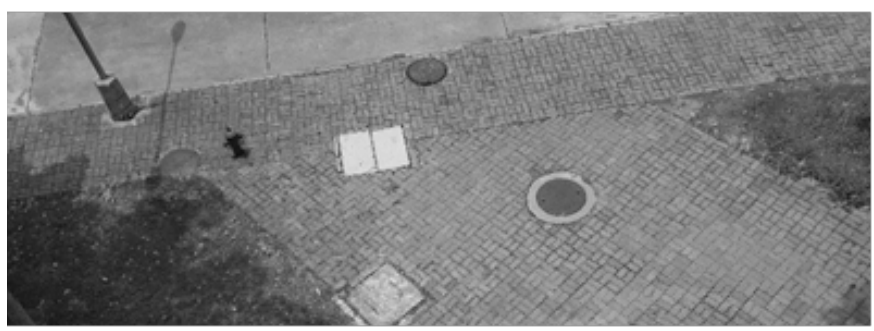

$\gg$ im = imread ('ph-101.png');

$\gg$ im $=$ rgb2gray $(i m)$;

$\gg \mathrm{dx}=[-1,0,1 ;-2,0,2 ;-1,0,1]$;

$\gg \mathrm{IX}=$ filter $2(\mathrm{dx}, \mathrm{im})$;

dy $=[1,2,1 ; 0,0,0 ;-1,-2,-1] ;$

$\gg I x=$ filter $2(d y, i m)$ :

$>$

$\gg \mathrm{Ix}=$ conv2 (double $(\mathrm{im}), \mathrm{dx}$, 'same") ;

$\gg \mathrm{Iy}=$ conv2 (double $(\mathrm{im}), \mathrm{dx}$, "same");

$\gg$

$\gg g=f$ special ('gaussian', [1 5],1.5)

$g=$

$$
\begin{array}{lllll}
0.1201 & 0.2339 & 0.2921 & 0.2339 & 0.1201
\end{array}
$$

> Step 2: Smooth space image derivatives (gaussian filtering)

$\gg \operatorname{Ix} 2=\operatorname{conv} 2(\mathrm{Ix} \wedge 2, \mathrm{~g}$, 'same') :

$\gg$ Iy2 $=\operatorname{conv} 2(\mathrm{Iy} \cdot \wedge 2, \mathrm{~g}$, 'same' $)$;

$\gg$ Ixy = conv2 (Ix.* Iy, g, 'same') ;

$\gg$ \& Step 3: Harris corner measure

$\gg$ harris $=\left(\operatorname{Ix} 2 \cdot{ }^{*}\right.$ Iy2 $\left.-\operatorname{Ixy} \cdot{ }^{\wedge} 2\right) . /(\operatorname{Ix} 2+\mathrm{Iy} 2)$ :

$\gg$ st Step 4: Find local maxima (non maximum suppression)

$\gg m x=$ ordfilt2(harris, $15 \times \wedge 2$, ones (15)) ;

$\gg$

$\gg$ step 5: Thresholding

$>$ harris $=($ harris $=m \mathrm{mx})$ \& (harris $>1000)$;

> imshow (im) :

Figure 10 Computing image gradients.

\section{Conclusion}

This paper proposes a preliminary choosing and design of a VTOL unmanned aerial system for real-time remote sensing of landscapes (RSL). Analysis of most existing UAS systems, suitable for solving RSL tasks allow determined which elements of the system will be 
manufactured by designer and which will be procured with estimated cost, from alternative outside contractors. The preliminary design phase achieves with an all-inclusive design meaning of the system with its interfaces and a system requirement. In the proposed approach, we use as more as possible typical, commercial of-the-shelf components that can be used to this type of system manufacture.

On the basis of design constraints and main steps of preliminary design proposed VTOL configuration. As a rational example of VTOL unmanned aerial system selected for, was demonstrated practical example on basis of micro-UAS quadrotor. Add MATLAB experiments with image features such as Harris Corners can serve as a compact image representation useful for task such as image matching, computing image statistics, 3D model estimation and video tracking during remote sensing of landscapes. To guarantee control during all flying stages, a distance acquisition system using sonic ranging sensors was choosing, installed and tested. So, proposed technique allows effectively made preliminary design of a VTOL unmanned aerial system for real-time remote sensing of landscapes.

\section{Acknowledgments}

None.

\section{Conflicts of interest}

Author declares that there is no conflict of interest.

\section{References}

1. Adams JB, Gillespie AR. Remote Sensing of Landscapes with Spectral Images: A Physical Modeling Approach. Cambridge University Press; 2006. 379 p.
2. Weng Q. Remote Sensing and GIS Integration: Theories, Methods, and Applications. McGraw-Hill Companies; 2010. 433 p.

3. Krishna KR. Agricultural Drones: A peaceful Pursuit. Apple Academic Press Inc: Oakville, Canada; 2018. 413 p.

4. Papa U, Ponte S. Preliminary Design of an Unmanned Aircraft System for Aircraft General Visual Inspection. Autonomous Control of Unmanned Aerial Vehicles, edited by V Beccerra. MDPI: UK; 2019. 190-204 p.

5. Austin R. Unmanned Aircraft Systems: UAVs Design, Development and Deployment. John Wiley \& Sons Ltd; 2010. 365 p.

6. Valavanis KP. Introduction. In Advances in Unmanned Aerial Vehicles. State of the Art and the Road to Autonomy, Springer: Dordrecht, The Netherlands; 2007. 3-13 p.

7. Bouabdallah S, Siegwart R. Design and Control of a Miniature Quadrotor. In Advances in Unmanned Aerial Vehicles. State of the Art and the Road to Autonomy, Valavanis, Springer: Dordrecht, The Netherlands; 2007. $171-210 \mathrm{p}$.

8. Schmidt MD. Simulation and Control of a Quadrotor Unmanned Aerial Vehicle. Ph.D. Thesis, College of Engineering, University of Kentucky: Lexington, Kentucky; 2011.

9. Prior SD. Optimizing small multi-rotor unmanned aircraft: A practical design guide. CRC Press; 2019. 365 p.

10. DJI Drone Motor Brushless DC Motor NFP-BL-2204.

11. Austin R. Unmanned Aircraft Systems: UAVs design, development and deployment. Wiley; 2010. 365 p.

12. HS700D FPV Drone with GPS

13. HS700 Ophelia vs HS700D Ophelia: What's The Difference?.

14. Dorhea Raspberry Pi4B3B+ Camera Module. 